\title{
La investigación-acción: una propuesta para la formación y titulación en las carreras de Educación Inicial y Primaria de una institución de educación superior privada de Lima
}

\author{
LIZA CABRERA MORGAN" \\ Pontificia Universidad Católica del Perú - Perú \\ Recibido el 24-02-2016; primera evaluación el 09-05-2017; \\ segunda evaluación el 22-05-2017; aceptado el 12-06-2017
}

\section{Resumen}

El presente ensayo busca socializar la propuesta de trabajo en torno al desarrollo de la investigación-acción en el proceso de titulación de las carreras de Educación Inicial y Primaria de una institución de educación superior privada de Lima, en el marco del proceso de implementación del nuevo plan curricular iniciado el 2013. En ese sentido, se resaltan las características de este tipo de investigación que se han encarnado en la práctica, el proceso que se ha asumido para su aplicación, así como los logros, las dificultades y los aspectos sobre los cuales se requiere continuar trabajando. Finalmente se proponen algunas reflexiones para la consolidación de este tipo de investigación en la estructura académica y organizativa de dicha institución, que pueden resultar útiles para aquellas instituciones de educación superior interesadas en aplicar este enfoque de investigación.

Palabras-clave: Investigación-acción, investigación educativa, formación docente, formación de profesores, formación de pedagogos, formación inicial de profesores.

\footnotetext{
Licenciada en Educación Primaria por la Facultad de Educación de la PUCP (1990). Máster en Enseñanza y Aprendizaje Abiertos y a Distancia, Universidad Nacional de Educación a Distancia (UNED) y Cátedra UNESCO (2006). Diplomada en "Diseño y Desarrollo Curricular" por la Universidad Católica de Uruguay en Convenio con la Oficina Internacional de Educación (OEI) y la Unesco. Docente auxiliar de la Facultad de Educación de la Pontificia Universidad Católica del Perú (PUCP). Miembro del Comité Directivo de la Maestría en Integración e Innovación Educativa de las Tecnologías de la Información y la Comunicación (TIC). Excoordinadora de la Práctica Pre-profesional en la Especialidad de Educación Primaria en la Facultad de Educación de la PUCP. Contacto: lcabrer@pucp.edu.pe
} 
Action research: A proposal for training and titration in the careers of Initial and Primary Education of a private higher education institution in Lima

\section{Abstract}

The goal of this essay is to share the project proposal about the use of the actionresearch approach in the degree completion award process for students in the Pre-school and Primary Education specialization in a private higher education institution of Lima. This is done within the framework of the implementation process for the new curriculum plan initiated in 2013. In that sense, we highlight the characteristics of this type of research that have been embodied in practice, the process followed for its application, as well as achievements, difficulties and the aspects that we still need to work out. Lastly, some thoughts are proposed to consolidate this type of research in the academic and organizational structure of this institution, which could prove useful for higher education institutions interested in the implementation of this research approach.

Keywords: Action-research, educational research, teacher education, teacher training, training of educators, pre-service teachers.

A pesquisa-ação: uma proposta de capacitação e titulação nas carreiras de Educação Inicial e Primária da instituição de ensino superior privado em Lima

\section{Resumo}

O presente ensaio tem como objetivo socializar a proposta de trabalho ao redor do desenvolvimento da Investigação- Ação no processo de concessão de licenciatura em docência dos educação infantil e anos iniciais do ensino fundamental da instituiçáo de ensino superior privado em Lima, dentro do contexto do processo de implementação do novo plano curricular iniciado em 2013. Nesse sentido se ressaltam as características deste tipo de investigação que foram adotadas na prática, o processo que se assumiu para sua aplicação, assim como os resultados, as dificuldades e os aspectos sobre os quais se requer continuar trabalhando. Finalmente, se propóe algumas reflexôes para a consolidação deste tipo de investigação na estrutura acadêmica e de organização da instituição, que podem resultar úteis para aquelas instituiçóes de educaçáo superior interessadas em aplicar este enfoque de investigação.

Palavras-chave: Investigação-ação, investigação educativa, formação docente, formação de professores, formação de pedagogos, formação inicial de professores. 


\section{¿En qué contexto curricular se propone la investigación-acción en las carreras de Educación Inicial y Primaria de una institución de educación superior privada de Lima?}

La institución mencionada, a través de su Facultad de Educación, viene implementando desde inicios de 2013 un nuevo plan curricular en las carreras de Educación Inicial y Primaria, sustentado en las necesidades y demandas del contexto local, global e institucional, así como en un conjunto de principios y opciones teóricas que se expresan en las siguientes características:

- Construcción social sustentada en una permanente reflexión y deliberación sobre la práctica para el desarrollo humano.

- Orientado al desarrollo humano en el marco de formación de valores.

- En búsqueda de la transformación del hombre y la sociedad.

- Formación para la diversidad, el diálogo, la autonomía y el compromiso con los otros.

- Construcción activa del conocimiento.

- Orientado al protagonismo del estudiante.

- Donde el formador sea orientador, facilitador y motivador de procesos educativos.

- Interdisciplinario, orientado a la práctica, a la investigación y al uso pertinente de las TIC.

Dicho plan desde un enfoque práctico, reflexivo y socio-crítico, busca formar profesionales que sean capaces de comprometerse con el desarrollo integral de los niños y las niñas y con la construcción de una sociedad justa y democrática, asegurando en todo momento una relación respetuosa con el medio ambiente y el desarrollo sostenible.

En tal sentido, se asume el currículo como un espacio de deliberación, diálogo democrático y reflexivo, y compromiso con la realidad educativa a partir de la contrastación permanente entre la teoría y la práctica. La práctica se convierte en el espacio privilegiado, donde los estudiantes integran y consolidan su conocimiento pedagógico y generan procesos cíclicos de acción-reflexión-innovación.

En concreto, la propuesta de formación se organiza en cuatro áreas curriculares, que son las mismas que organizan nuestro perfil del egresado y se desarrolla en diez semestres académicos. Dichas áreas son: 1) Desarrollo personal y profesional, 2) Investigación y Práctica educativa, 3) Gestión de organizaciones educativas y Liderazgo, y 4) Procesos de enseñanza y aprendizaje. 
El área de "Investigación y Práctica Educativa" constituye el área troncal, transversal e integradora de las demás áreas, por tanto, del proceso de formación; dicha área está conformada por diez cursos que se distribuyen a lo largo del Plan de Estudios, uno en cada semestre académico, de modo de desarrollar en forma progresiva habilidades, destrezas y actitudes tanto para la investigación como para el trabajo pedagógico en diversos escenarios educativos (formales, no formales y alternativos). En la figura 1 se aprecia la secuencia de dichos cursos.

Figura 1. Línea de Investigación y Práctica Educativa

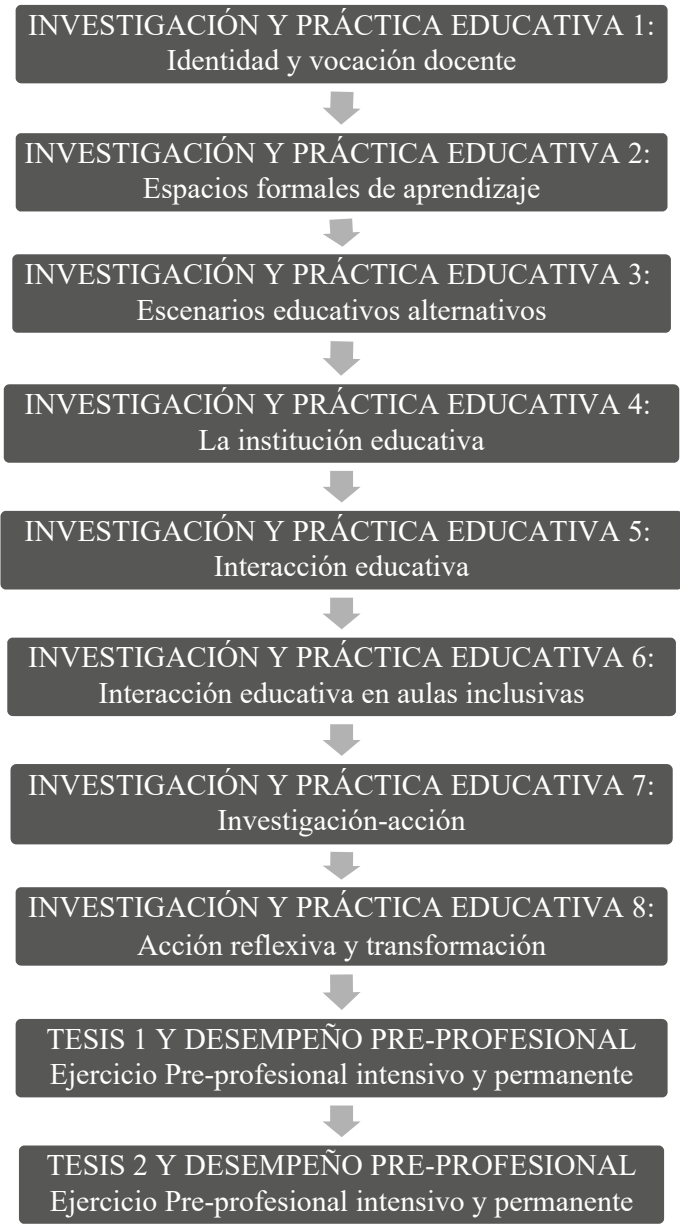

Fuente: Elaboración propia a partir del Plan de Estudios de la institución educativa en mención. 
En ese sentido, todos los esfuerzos se encaminan a un avance gradual de lo simple a lo complejo, del contexto personal y particular del estudiante a diversos contextos o escenarios propios de la profesión; asimismo, del simple rol de observador al de un activo artífice de los aprendizajes en el aula, pasando en este proceso por los roles de observador participante, ayudante y practicante, tal como se muestra en la figura 2 .

\section{Figura 2. Rol del estudiante en su proceso de formación}

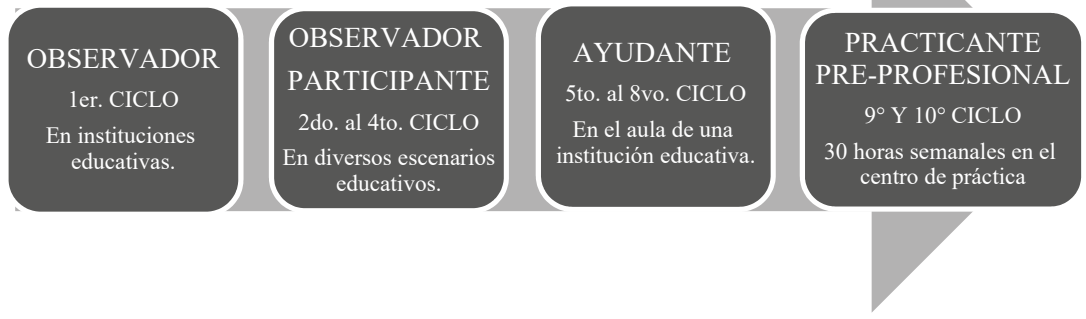

Fuente: Adaptación a partir del Plan de Estudios de la institución educativa en mención.

Hay que destacar que la participación del estudiante en diversos escenarios educativos resulta indispensable, desde el inicio de la carrera, pues ello tiene significativo impacto en la afirmación de su vocación docente, en su compromiso social y en la mejora constante de su desempeño. Algunos de estos escenarios son: las escuelas, las asociaciones culturales, los centros comunitarios, las instituciones científicas, artísticas, de salud y de investigación educativa, los organismos no gubernamentales, los medios de comunicación, entre otros.

Se trata de asegurar a través de todos los cursos del Plan de Estudios y, en particular, de esta área troncal, una propuesta de práctica educativa secuenciada, progresiva y articulada con el trabajo de investigación, que posibilite al estudiante disponer de un escenario para construir y contrastar conocimientos, reconocer y reflexionar sobre problemas educativos reales, diseñar y aplicar propuestas de acción y/o mejora; en definitiva, formar y consolidar habilidades y actitudes para la docencia.

En el marco expuesto, la investigación-acción representa una herramienta de aprendizaje fundamental, un medio a través del cual el futuro docente puede desarrollar el perfil propuesto por la institución formadora, en su confrontación permanente entre la teoría y la práctica, el ejercicio reflexivo 
y crítico, y la asesoría y acompañamiento de un equipo de docentes. Según Restrepo, "La investigación-acción es un instrumento que permite al maestro comportarse como aprendiz de largo alcance, aprendiz de por vida, ya que le enseña como aprender a aprender, cómo comprender la estructura de su propia práctica y cómo transformar permanente y sistemáticamente su práctica pedagógica” (2003, p. 7).

Si bien la formación en habilidades y actitudes investigativas se da a lo largo del plan de estudios, en adelante nos referiremos estrictamente a aquellos proyectos de investigación-acción que los estudiantes desarrollan, en el último año de formación (dos semestres académicos), en el contexto de la práctica pre-profesional intensiva y permanente, como responsables o corresponsables de una aula, a través de los cursos "Tesis y Desempeño Pre-profesional 1 y 2". Según la finalidad de estos cursos, y de acuerdo a las exigencias legales de las prácticas pre-profesionales en nuestro país, todos los estudiantes de educación permanecen en una institución educativa (escuela), durante 30 horas semanales, de lunes a viernes, en un lapso de seis horas diarias, desde el inicio del horario escolar. Empiezan desarrollando solo algunas actividades pedagógicas y progresivamente van incrementando su participación, en la medida que van demostrando sus competencias docentes. En este escenario concentran sus esfuerzos no solo en las tareas propias del ejercicio docente en aula, encaminadas a facilitar el aprendizaje activo, participativo y creativo de los alumnos; sino en el diseño y puesta en marcha de un proyecto de investigación para efectos de su titulación. Por las tardes retornan al campus universitario para participar en otros cursos previstos en el plan de estudios.

Cabe señalar que los alumnos pueden optar libremente entre dos modalidades para obtener el título de Licenciado en Educación, ya sea mediante un proyecto de investigación o un proyecto de innovación; sin embargo, este proyecto deben realizarlo obligatoriamente en el marco del último año de formación. Esta estrategia está permitiendo elevar el índice de graduación de nuestros egresados. Existe otra modalidad de titulación, que es la sistematización de una experiencia profesional, pero esta se dirige a egresados con varios años de experiencia profesional en el campo educativo. Justamente en el marco del proyecto de investigación existen valiosas experiencias de alumnos del último año de formación, que han puesto en marcha proyectos bajo el enfoque de la investigación-acción. A continuación compartiremos lo avanzado hasta el momento en este tipo de investigación. 


\section{¿Cómo concebimos la investigación-acción?}

Partimos por reconocer que este tipo de investigación representa una alternativa viable para dar respuesta a los problemas cotidianos y acuciantes que experimentan los docentes en el ejercicio de su tarea, con el fin de producir mejoras en sus prácticas educativas. Según Lewin (1946), su creador, y otros autores que desarrollaron este tipo de investigación como Carr y Kemmis (1988), dicha investigación implica la visión dialéctica entre la investigación y la acción, de modo que ambos procesos quedan integrados y complementados a través de fases cíclicas que proponen planificar, actuar, observar y reflexionar. Se trata de una investigación transformadora de la realidad y la enseńanza, una actividad cuestionadora, reflexiva, contextualizada, participativa que articula la teoría y la práctica, el conocimiento y la acción.

Ha resultado iluminador para nuestro trabajo la siguiente definición de investigación-acción, propuesta por Kemmis y McTaggart, que sigue vigente en nuestros días; en ella se destaca la intención de un cambio y mejora de la acción educativa, con una visión dinámica de la realidad, así como la participación activa y comprometida de un conjunto de actores en pos de una tarea de cambio social. Veamos: "Una forma de indagación introspectiva colectiva emprendida por participantes en situaciones sociales, con objeto de mejorar la racionalidad y la justicia de sus prácticas sociales o educativas, así como su comprensión de esas prácticas y de las situaciones en que estas tienen lugar» (1988, p. 9).

Estos mismos autores sostienen que es posible mejorar la educación cambiando las prácticas, siendo el análisis reflexivo y la acción sobre la práctica el medio idóneo para generar los cambios. En este proceso, Suárez (2002) señala que es fundamental recoger evidencias de lo que sucede y de los cambios que se van produciendo, a través de diversas técnicas aplicadas en forma sistemática.

Desde nuestra experiencia como docentes, apreciamos cómo este tipo de investigación hace posible que la práctica y la teoría se encuentren en un espacio de diálogo común, donde los estudiantes van contrastando los datos de la realidad con los conceptos de base aprendidos en su proceso de formación y estos últimos retornan al escenario educativo para dar un nuevo sentido a su práctica o enriquecerla. Es pues, el centro de prácticas, el aula de clases, un laboratorio donde se someten a prueba hipótesis y propuestas, donde el docente, en este caso, el alumno practicante, tiene la responsabilidad y el compromiso de impulsar dicho proceso, con la asesoría directa del docente responsable del aula y del docente asesor de la institución formadora. 
Podemos decir que hay un valor agregado en el desarrollo de los proyectos de investigación-acción durante las prácticas preprofesionales, pues al mismo tiempo que el futuro docente asume la investigación como parte de su tarea profesional, preparándose para poner en marcha propuestas de mejora e innovación pedagógica, en el marco de una práctica reflexiva; también, al término de su formación tiene la posibilidad de sustentar su trabajo de investigación para obtener el título de Licenciatura en Educación.

\section{¿Qué características destacamos en este tipo de investigación?}

Las características que asignamos a la investigación-acción, son las que destacan sus principales representantes: Lewin (1946), Kemmis y McTaggart (1988), Lomax (1990) Zuber-Skerritt (1992) y Elliot (1993), todos ellos citados por Latorre (2007). A continuación nos referiremos a ellas.

- Es un proceso participativo y colaborativo, pues involucra a los diversos miembros de la comunidad educativa en los cambios que se buscan generar, y se espera de todos ellos compromiso social.

- Es un proceso democrático basado en el diálogo, que respeta los aportes de todos los actores de la comunidad.

- Es un proceso político porque involucra cambios que afectan a las personas y a sus organizaciones.

- Dicho proceso está orientado a producir mejoras en las propias prácticas en el aula y en las concepciones y actitudes de las personas involucradas, en particular del docente y de los alumnos.

- Es una práctica reflexiva, pues los profesores, en nuestro caso, alumnos practicantes, evalúan sus propias cualidades, tal como se manifiestan en sus acciones.

- Sigue un proceso circular y flexible que comprende varias fases: planificación, acción, observación y reflexión.

- Es un proceso sistemático de aprendizaje orientado a la praxis, que requiere llevar un seguimiento y registro de las reflexiones y evidencias de los avances.

- Somete a prueba las prácticas, ideas, suposiciones y juicios de las personas involucradas en la investigación, con el fin de enriquecer el saber profesional y la propia práctica. 
- Integra la teoría en la práctica, es decir, induce a teorizar o construir el conocimiento por medio de la práctica.

- De lo anterior se desprende que, es crítica y transformadora pues busca actuar sobre necesidades sentidas, a través de un plan de intervención que tenga impacto en la calidad de los procesos y en las mismas personas.

\section{¿Qué procesos asumimos en el desarrollo de la investigación-acción?}

Los proyectos de investigación-acción desarrollados por los estudiantes durante los años 2014 a 2016, han sido orientados a partir de lo propuesto por McKernan (2001), donde se inicia el trabajo con el reconocimiento, análisis y documentación de una situación que ocurre en las aulas y que se desea cambiar o mejorar, y a partir de ella se diseña y pone en marcha un plan de intervención en el que también se observa, se reflexiona, se analiza lo que ocurre, y se evalúan los cambios que se producen. De este modo, se abre la posibilidad al estudiante-investigador de volver a plantear un nuevo ciclo de investigación y acción. Veamos con exactitud lo que propone este autor:

La investigación-acción es el proceso de reflexión por el cual en un áreaproblema determinado, donde se desea mejorar la práctica o la comprensión personal, el profesional en ejercicio lleva a cabo un estudio -en primer lugar-, para definir con claridad el problema; en segundo lugar, para especificar un plan de acción- que incluye el examen de hipótesis por la aplicación de la acción al problema. Luego se emprende una evaluación para comprobar y establecer la efectividad de la acción tomada. Por último, los participantes reflexionan, explican los progresos y comunican estos resultados a la comunidad de investigadores de la acción. La investigación-acción es un estudio científico autorreflexivo de los profesionales para mejorar la práctica (p. 25).

Del mismo modo, en concordancia con Latorre (2007), asumimos que la investigación-acción es un espiral autorreflexivo, donde las fases de planificación, acción, observación y reflexión resultan vitales, y se dan en forma flexible y cíclica durante todo el proceso.

En el siguiente diagrama (figura 3) se puede apreciar en detalle las fases o etapas que asumen los estudiantes para llevar a cabo sus proyectos de investigación-acción; hay que recordar que estas etapas no son lineales: 
Figura 3. Proceso para el desarrollo de la Investigación-acción

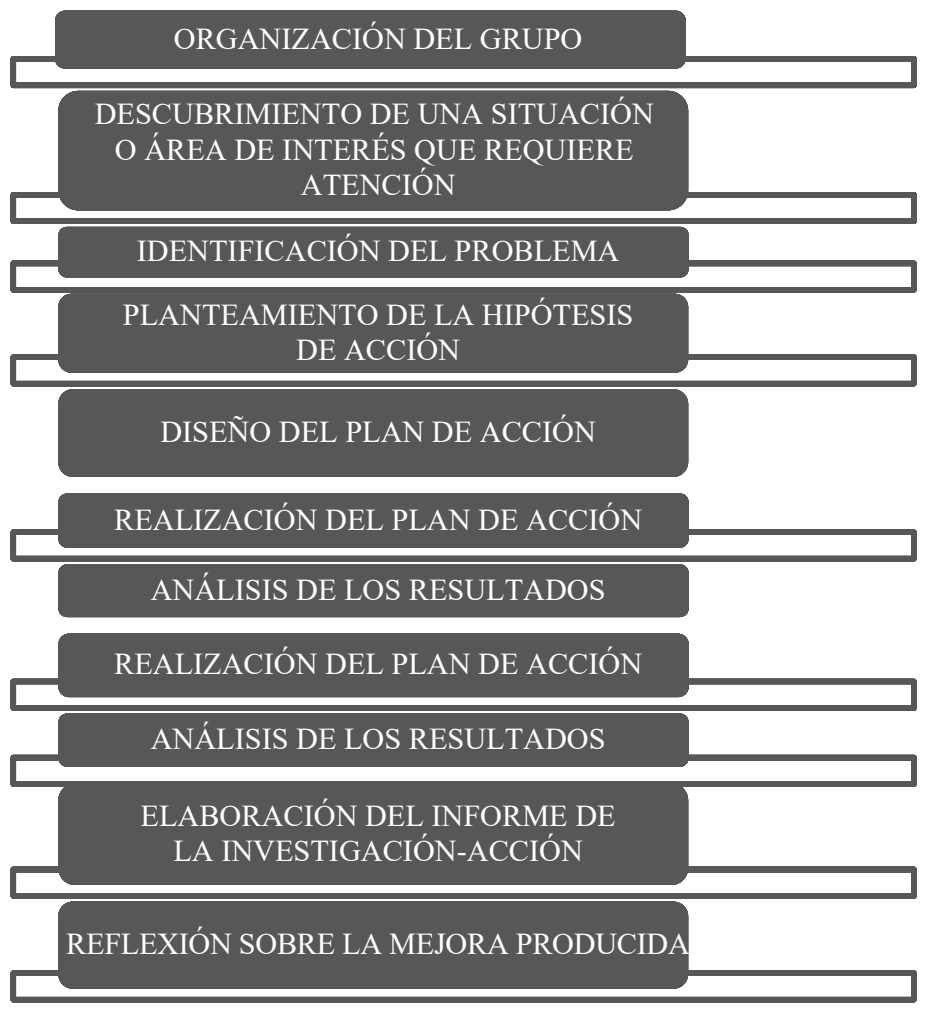

Es en el curso "Tesis 1 y Desempeńo Pre-profesional", en el marco de la práctica pre-profesional intensiva, durante el noveno ciclo de estudios, que los estudiantes que eligieron el proyecto de investigación-acción como modalidad de titulación, realizan una aproximación diagnóstica en el contexto de una institución educativa (escuela) y de un aula de clases, a partir de la cual priorizan una situación de su práctica que requiere mejora, atención o consolidación, con participación de la profesora responsable del aula y, en algunos casos, con el aporte de las autoridades de la institución. Dicha situación no solo debe estar vinculada al aprendizaje de los alumnos y a la enseńanza del docente (alumno practicante), sino que debe resultar viable de asumir en el marco temporal del ańo de práctica pre-profesional, que implica treinta horas semanales (seis horas diarias) de participación en el centro de práctica. Es así que los estudiantes trabajan entusiasmados para documentar y reflexionar en torno a dicha situación, confirmando su significatividad, pertinencia y viabilidad. 
¿Qué es lo característico en este tipo de investigación? Según Elliot (2010):

Mientras el científico natural y el del comportamiento comienzan con un problema teórico definido en su propia disciplina, el investigador en la acción comienza con un problema práctico. Pero en determinado sentido, el problema de este último es teórico también. Surge en la experiencia de la inadecuación entre sus teorías prácticas y la situación a la que se enfrenta. La única diferencia entre el práctico y el científico natural o comportamental consiste en que la teoría del primero con frecuencia está incluida en su propia práctica [...] (p. 97).

Una vez definida y documentada la situación sobre la que se va a trabajar, los estudiantes reflexionan con el apoyo de la profesora responsable del aula, en torno a la forma de abordar o atender dicha situación, definiendo así el plan de intervención, también llamado plan de acción. Este es el momento para diseñar y presentar formalmente a la institución formadora, para efectos de la titulación, el Plan de Investigación con el proyecto elegido. En dicho Plan se precisa y justifica el problema de la investigación, sus objetivos y la hipótesis de acción; también, se explica el enfoque metodológico, las técnicas y los instrumentos a aplicar, así como los informantes; finalmente, se presenta el cronograma de trabajo y la bibliografía de referencia.

A partir de este Plan de Investigación, los estudiantes concentran sus esfuerzos durante el noveno ciclo de estudios, en dos tareas centrales: primero, delimitar y desarrollar los supuestos teóricos vinculados con la situación identificada, los cuales permitirán iluminar la acción o intervención posterior; y segundo, diseñar el plan de acción para atender dicha situación. Esto último incluye precisar cómo se intervendrá, es decir, qué se va a hacer, con qué medios y recursos y bajo qué cronograma de trabajo. Estas tareas significan para el estudiante un proceso de contrastación entre la investigación bibliográfica realizada y la reflexión sobre los datos concretos de la realidad; asimismo, un diálogo oportuno y cercano con los actores de la institución para asegurar la pertinencia y viabilidad del plan de acción. Cabe destacar que a lo largo del proceso de cada proyecto de investigación y de la práctica educativa en la escuela, cada estudiante recibe la asesoría y acompañamiento periódico de un docente designado por la institución formadora. En tal sentido, un docente tiene bajo su responsabilidad un número variable de estudiantes, dependiendo de la distribución de su carga docente, no pasando en ningún caso de cuatro asesorados bajo su responsabilidad Ya en el curso "Tesis 2 y Desempeńo Pre-profesional" que se desarrolla en el $10^{\circ}$ ciclo de estudios, los estudiantes implementan y ponen en marcha el plan de intervención diseñado, 
buscando producir mejoras en algún aspecto de su práctica educativa, que tenga repercusión directa en el aprendizaje de los alumnos a su cargo y en su propio desempeño docente. Hay que reiterar que dicho plan es compartido y enriquecido por la profesora responsable del aula y, en algunos casos, por las autoridades de la institución educativa.

Resulta de gran importancia el seguimiento que realizan los estudiantes de todas las acciones que comprende el plan de acción, para lo cual se han dispuesto determinadas técnicas e instrumentos. Se trata de reunir evidencias significativas en torno a las acciones desarrolladas y a los aprendizajes que se han producido en los sujetos que participan en la investigación, esto es, en los alumnos, el/la docente del aula y el estudiante-investigador. Esto puede suponer cambios en las percepciones y valoraciones de los sujetos participantes, el uso de nuevos conocimientos y técnicas, y/o el desarrollo de determinadas habilidades, destrezas o actitudes. Se puede decir que las evidencias recogidas permiten analizar en qué medida y de qué modo se ha logrado atender la situación inicialmente detectada. Aquí resulta prioritario asegurar durante el proceso los espacios de diálogo y reflexión entre los actores de la investigación mencionados, con el fin de generar conciencia y responsabilidad sobre lo que se va logrando, sobre el proceso vivido, así como sobre lo que piensan y sienten acerca de los cambios o mejoras que se han producido.

Las técnicas más usadas por los estudiantes-investigadores en este proceso de seguimiento han sido las observaciones, a través del diario de clase y la lista de cotejo; las entrevistas estructuradas o semiestructuradas, dirigidas a los nińos del aula y a la docente; el diálogo en pequeños grupos de alumnos con ayuda de una guía de preguntas; las encuestas, a modo de autoevaluaciones (cuestionarios), también dirigidas a los alumnos; y el análisis documental, esto es, la revisión de los trabajos producidos por los niños y niñas del aula.

Posterior a la aplicación del plan de intervención, los estudiantes proceden a la organización de la información en categorías, a través de cuadros, gráficos y mapas, con el fin de facilitar su posterior análisis e interpretación. Aún no se ha llegado a incorporar en esta fase del trabajo a los demás actores de la institución, debido al escaso tiempo del cual disponen los estudiantes para la realización de esta tarea.

Una vez analizada e interpretada la información, los estudiantes proceden a la redacción del informe final de la investigación-acción, con la asesoría permanente del docente-asesor de tesis y, de acuerdo a las orientaciones metodológicas y técnicas, que propone la institución formadora. En la figura 4 se muestra cómo se estructura dicho informe: 
Figura 4. Estructura del Informe Final de la investigación-acción

\begin{tabular}{|c|c|}
\hline $\begin{array}{l}\text { Componente del } \\
\text { Informe Final }\end{array}$ & Descripción \\
\hline Introducción & $\begin{array}{l}\text { Planteamiento y justificación del problema de investigación- } \\
\text { acción, motivaciones personales, aportes dela investigación-acción. }\end{array}$ \\
\hline $\begin{array}{l}\text { PARTE I: } \\
\text { Marco Teórico }\end{array}$ & Referentes conceptuales coherentes con el objeto de estudio. \\
\hline $\begin{array}{l}\text { PARTE II: } \\
\text { Diseño de Investigación }\end{array}$ & $\begin{array}{l}\text { Método de la investigación-acción. Contexto de la investi- } \\
\text { gación-acción. Plan de acción. Técnicas e instrumentos para } \\
\text { organizar y analizar la información. }\end{array}$ \\
\hline $\begin{array}{l}\text { PARTE III: } \\
\text { Análisis e interpretación } \\
\text { de los resultados }\end{array}$ & $\begin{array}{l}\text { Narración del proceso de investigación-acción vivido desde la } \\
\text { identificación del problema, el desarrollo del plan de acción, los } \\
\text { logros y dificultades encontrados. }\end{array}$ \\
\hline Lecciones aprendidas & $\begin{array}{l}\text { Reflexión sobre el proceso vivido: aprendizajes significativos en } \\
\text { torno a los cambios generados y a los problemas más sentidos. }\end{array}$ \\
\hline Bibliografía & $\begin{array}{l}\text { Listado de fuentes consultadas en la investigación-acción, } \\
\text { empleando normas APA (sexta edición). }\end{array}$ \\
\hline Anexos & $\begin{array}{l}\text { Instrumentos aplicados, cuadros, gráficos y otros documentos } \\
\text { complementarios relevantes. }\end{array}$ \\
\hline
\end{tabular}

Fuente: Síntesis del documento Modalidades de Titulación (2013) de la institución educativa en mención.

Se promueve que los estudiantes compartan los resultados del Informe Final con los actores participantes en la investigación, para lo cual se les sugiere una presentación ágil y motivadora de dichos resultados, destacando los procesos vividos, utilizando ayudas visuales que faciliten la comprensión de las mejoras que se han producido. Los trabajos producidos por los niños, así como las fichas de evaluación y autoevaluación empleadas para el seguimiento del plan de acción, resultan evidencias significativas, que permiten ilustrar el trabajo realizado en el aula.

Cabe señalar que en los dos cursos comentados, "Tesis y Desempeño Pre-profesional 1 y 2", se ha utilizado la asesoría individual y en pequeños grupos, por turnos, en forma periódica y permanente, para orientar el desarrollo de cada proyecto de investigación. El diálogo reflexivo y en momentos claves entre asesor y estudiante-asesorado, ha facilitado que este último pueda encontrar sus propias respuestas para avanzar en el desarrollo de su investigación, generar nuevas interrogantes, redefinir decisiones y confirmar la ruta de trabajo. Definitivamente, todo ello ha tenido impacto en la calidad de su estudio y en la consolidación de sus habilidades y actitudes investigativas y para la docencia. 


\section{¿Qué logros y dificultades hemos experimentado al aplicar la investigación- acción? ¿Por dónde continuar trabajando?}

Actualmente nos encontramos en el cuarto año de implementación de esta experiencia de investigación-acción, en el marco del nuevo plan curricular de las carreras de Educación Inicial y Primaria; sin embargo, hasta el momento se han desarrollado este tipo de investigaciones solo en la carrera de Educación Primaria. Hay que señalar que la modalidad y enfoque de investigación es producto del interés y decisión personal de cada estudiante. A continuación deseamos compartir algunas reflexiones en torno al trabajo realizado hasta el momento, en el contexto de los cursos aludidos (Tesis y Desempeño Preprofesional 1 y 2), con el fin de potenciar el uso de este enfoque de investigación como una modalidad alternativa de formación y titulación en las carreras de Educación; y asimismo, visualizar las mejoras que es necesario introducir para asegurar la calidad de los proyectos presentados por los estudiantes.

A partir de la revisión bibliográfica hecha en torno al tema de la investigación-acción y de la experiencia en la asesoría de este tipo de proyectos, deseamos proponer algunas reflexiones que nos permitan clarificar el lugar de este tipo de investigación en el proceso de formación de los futuros docentes en nuestra institución educativa.

- Los proyectos de investigación-acción constituyen una herramienta fundamental para asegurar la integración de la práctica educativa con la labor de investigación, como dos actividades medulares e indisolubles de la labor docente, y que son coherentes con el enfoque práctico y socio-crítico del nuevo plan curricular por el que hemos optado para la formación de docentes en nuestra institución. Sin embargo, somos conscientes que son los estudiantes quienes libremente deciden qué tipo de investigación diseñar y llevar a cabo, en función a sus intereses.

- Los proyectos de investigación-acción, a través de los planes de intervención o acción, representan excelentes oportunidades para asegurar un impacto directo en la propia actuación del estudiante como futuro docente y en los procesos de aprendizaje de los niños que atienden.

- Los proyectos de investigación-acción desencadenan la expectativa de los maestros y de las autoridades de la escuela al evidenciar su aporte en la mejora de los procesos de enseńanza y aprendizaje, particularmente, en la innovación de estrategias didácticas y en el uso de recursos para el aprendizaje. Hay que tener en cuenta que los estudiantes practicantes 
son, en muchos casos, dinamizadores de la renovación didáctica, pues llevan a la escuela nuevos conocimientos, técnicas y herramientas para el aprendizaje significativo, activo y participativo en diferentes áreas curriculares.

- Las instituciones educativas que comprenden la importancia del desarrollo de los proyectos de investigación-acción suelen mostrar motivación y compromiso para brindar los apoyos y/o recursos que faciliten su desarrollo y culminación. Por tanto, resulta indispensable, en una fase inicial, motivar e informar con pertinencia a las autoridades $\mathrm{y}$ al equipo docente de dichas instituciones.

- Uno de los beneficios de este enfoque de investigación, es que los estudiantes no necesitan buscar otros espacios físicos y temporales para la realización de sus estudios orientados a la titulación. El aula de clases donde realizan su práctica pre-profesional se convierte en el escenario idóneo donde descubren y analizan las necesidades o situaciones que son de interés o requieren atención, y donde proyectan las acciones de intervención, en el marco del trabajo curricular que tienen asignado.

- En este contexto, resulta fundamental que los estudiantes-practicantes puedan asumir una actitud reflexiva, crítica, abierta y flexible, en todas las etapas del proyecto de investigación-acción, que les permita recoger las voces de los actores de la comunidad educativa, para tomar en todo momento decisiones pertinentes y oportunas, que garanticen el éxito de sus esfuerzos.

- Tiene especial relevancia que los estudiantes en su práctica cotidiana en el aula promuevan dicha actitud reflexiva entre los nińos, cuando dialogan y reflexionan sobre lo aprendido y sobre las dificultades experimentadas en la ruta de sus aprendizajes.

En las líneas siguientes haremos una revisión crítica de las dificultades que se han documentado frente a la implementación de los proyectos de investigación-acción, con el fin de aproximarnos posteriormente a algunas soluciones. En tal sentido, recogeremos algunos de los aportes de Balcazar (2003):

- En primer lugar la actitud de "arrogancia intelectual" (p.71) del investigador cuando se enfrenta a un nuevo contexto, en nuestro caso, la escuela y el aula en particular, con convicción de que tiene ya todas las respuestas entre manos. Esta actitud no genera la confianza necesaria 
para desarrollar las relaciones interpersonales que permitan la motivación y compromiso de la comunidad.

- La falta de visión o conciencia compartida entre los investigadores y los miembros de la comunidad (en este caso, la comunidad educativa), lo que les impide visualizar y asumir que ellos pueden ser gestores de sus propios procesos de cambio y mejora. Esto debe movilizar a todos los miembros de la comunidad a trabajar sobre sus miedos, inseguridades, ignorancia y la falta de confianza en sus propias capacidades.

- La falta de recursos materiales y humanos para poner en marcha los proyectos también es una dificultad, y puede limitar la motivación de sus participantes, así como la continuidad de las acciones del plan de intervención o acción.

- La falta de tiempo para llevar a término el proceso de intervención, sobre todo si se trata de producir cambios sustantivos en los procesos o en las personas. Normalmente cuando los proyectos implican cambios en las disposiciones, habilidades y actitudes de las personas, es necesario considerar tiempos considerables de actuación y práctica repetida, lo cual puede disminuir la motivación de los participantes.

Según Balcazar (2003, p. 73), "Esto puede ser un problema para profesionales que son presionados por el contexto académico para producir publicaciones a corto término o facilitar proyectos de tesis de estudiantes, quienes generalmente solo tienen de seis a doce meses para completar su investigación". Sin embargo, lo importante es ir experimentando progreso en el proceso y que los esfuerzos de cambio desplegados estén dando sus frutos.

A continuación nos permitimos añadir algunas otras reflexiones en torno a las dificultades en la implementación de los proyectos de investigación-acción, que a nuestro juico resultan relevantes:

- En primer lugar, resulta complicado asegurar que todo el equipo de profesores de la institución formadora, a cargo del proceso de asesoría y seguimiento de los proyectos de investigación-acción, tenga una formación común sobre dicho enfoque de investigación, debido a que este equipo va cambiando año a año según las cargas lectivas de cada docente. En ese sentido, resulta recomendable continuar implementando espacios de capacitación en temas claves y de socialización en 
torno a las preocupaciones e interrogantes que van surgiendo al abordar este tipo de investigación, de modo de encontrar estrategias de trabajo pertinente y eficiente para consolidar este tipo de investigación en la institución formadora.

- Del mismo modo, en la búsqueda de una visión compartida sobre el tema de investigación-acción resulta complicado involucrar a los docentes y a las autoridades que laboran en las escuelas sobre la naturaleza y exigencias de este enfoque de investigación, pues se requieren variadas acciones de información, motivación y toma de decisiones en torno a las rutas y los calendarios de trabajo para el diseño y desarrollo de los proyectos de investigación-acción. Sin embargo, no se trata de una falta de voluntad política sino de espacios reales para el trabajo compartido, en medio de las múltiples tareas que deben desempeñar los docentes y las autoridades de las escuelas.

- Teniendo en cuenta que los primeros proyectos de investigaciónacción, fueron desarrollados por una promoción de estudiantes que no llevó sistemáticamente todos los cursos correspondientes a la línea de "Investigación y práctica educativa" del nuevo plan curricular, dichos estudiantes no contaron con las habilidades y conocimientos necesarios para el desarrollo de este enfoque de investigación, lo cual exigió un proceso de soporte, asesoría y seguimiento más detenido por parte del equipo docente. En ese sentido, es recomendable evaluar si el estudiante que elige realizar el proyecto de investigación-acción cuenta con los prerrequisitos necesarios para asumir las exigencias de dicho proyecto de investigación.

En función a las dificultades antes comentadas, nos proponemos compartir algunas ideas que han surgido de la reflexión teórica y de la misma práctica, y que nos han permitido encaminar valiosas alternativas de solución en el desarrollo de los proyectos de investigación-acción. Estas son:

- El equipo docente de la institución formadora, a cargo de los proyectos de investigación-acción, debe continuar en procesos de capacitación y espacios de socialización, orientados a consolidar conceptos y técnicas y compartir hallazgos y preocupaciones en la aplicación de este enfoque, de modo de responder eficientemente a las necesidades del sistema de asesoría y acompańamiento a los estudiantes. 
- Se requiere continuar fortaleciendo los vínculos entre la institución formadora de docentes y las escuelas que actúan como centros de práctica para nuestros estudiantes, de modo de apropiarnos de una visión compartida en torno a la naturaleza, exigencias y beneficios del desarrollo de proyectos de investigación-acción en las aulas. Esto fortalecerá la articulación entre la teoría y la práctica que es una de las características esenciales de nuestra propuesta de formación docente.

- Asimismo, necesitamos continuar fortaleciendo a través de los cursos de la línea de Investigación y Práctica Educativa de nuestro plan de estudios, los prerrequisitos que los alumnos necesitan, para asumir el diseño y desarrollo de los proyectos de investigación-acción. Dicho de otro modo, requerimos asegurar el desarrollo pertinente y gradual de habilidades, destrezas, actitudes y conocimientos en torno a este enfoque de investigación, desde el inicio de la carrera, de modo que los estudiantes lleguen al último año de formación preparados con rigurosidad para enfrentar los retos de este tipo de investigación. En ese sentido, es indispensable revisar y consolidar la secuencia de habilidades investigativas y para la práctica pedagógica, que se diseñó a partir del nuevo plan de estudios, con el fin de orientar el desarrollo de todos los cursos del plan de formación.

- De la misma manera, los docentes de la institución formadora necesitamos continuar fortaleciendo nuestro desempeño en torno al enfoque de la investigación-acción, a partir de proyectos sobre nuestra propia práctica educativa. Esto será una oportunidad propicia para continuar construyendo nuestro saber profesional.

- Dado que la institución formadora propone diversas vías para el proceso de titulación en las carreras de Educación Inicial y Primaria, se requiere motivar a los estudiantes, para que encuentren los beneficios de realizar estudios en el marco de la investigación-acción.

- Finalmente, resulta conveniente, a la luz de la experiencia, elaborar una guía de trabajo para el diseño y desarrollo de proyectos de investigación-acción, que facilite al estudiante trabajar en forma independiente, y por tanto, aprovechar en forma más eficiente el tiempo dispuesto para la asesoría personalizada, es decir, el encuentro entre el docenteasesor y el estudiante-investigador. 


\section{Algunas reflexiones finales}

Las páginas anteriores nos permiten plantear, ahora, algunas reflexiones para quienes estén interesados en emprender proyectos de investigación-acción, en el contexto de la propuesta de formación docente universitaria. En primer lugar, debemos señalar que la investigación-acción exige al docente-investigador cambiar ciertos paradigmas, pues no se trata de un trabajo de laboratorio, ni de arribar a la comprobación de datos o comportamientos; se trata de una inserción en una realidad educativa determinada, desde donde se analiza las prácticas, se contrasta con la teoría y se busca cambios o mejoras en el ámbito educativo. Según Elliot (2010, p. 24), este tipo de investigación en las escuelas "analiza las acciones humanas y las situaciones sociales experimentadas por los profesores como: (a) inaceptables en algunos aspectos (problemáticas); (b) susceptibles de cambio (contingentes); (c) que requieren una respuesta práctica (prescriptivas)." En cualquiera de los casos, el docente se compromete con sus propias prácticas para tener impacto en la calidad de los aprendizajes y en el desarrollo de las personas.

En segundo lugar, la investigación-acción busca actuar sobre las situaciones reconocidas para producir una mejora, considerando el punto de vista de quienes participan e interactúan en la situación reconocida. Si bien hay diversos modelos en este tipo de investigación, todos ellos tienen aspectos en común, siendo lo más significativo "el interés por intervenir en la praxis con el fin de mejorarla” (Pérez Serrano, 1990, p. 96).

Dicho lo anterior, nos toca desde nuestro quehacer docente, no solo motivar y orientar a nuestros estudiantes para que investiguen sobre sus propias prácticas, sino mirar nuestro propio escenario de trabajo, reconocer lo que hacemos y cómo lo hacemos, con el fin de descubrir qué es aquello en lo cual podemos producir mejoras. En este ejercicio hay que tener claro que, si bien partimos de una pregunta orientadora (problema de investigación), que se convierte en el norte de nuestra investigación, es necesario estar abiertos a nuevas interrogantes que pueden capturar nuestra atención y dinamizar nuestra acción. Lo importante entonces es, seguir haciendo preguntas y continuar aprendiendo de ellas. De esta manera, la investigación-acción se convierte en un proceso dinámico y flexible, que exige en todas sus fases: observación, reflexión, planeación y actuación.

Para asumir los retos que plantea este tipo de investigación, los docentes tenemos la exigencia de asegurar una formación sólida y de renovación constante a través de espacios de socialización, talleres $\mathrm{u}$ otros, que nos permita motivarnos y motivar a nuestros estudiantes para la transformación de nues- 
tras prácticas y para el compromiso con el desarrollo de nuestras instituciones y la educación de nuestro país. En ese sentido, prepararnos en el campo de la investigación-acción exige apropiarnos de nuevos modos de hacer investigación; esto es, ejercitarnos en procesos cíclicos de observación, reflexión y acción, que nos permita motivar y dinamizar las transformaciones en la dinámica de nuestro ejercicio profesional y en el desempeño de nuestros estudiantes.

Por otro lado, necesitamos fortalecer el sistema de asesoría individual a los alumnos, ampliando el tiempo de dedicación del docente a cada alumno, con el fin garantizar la calidad de los proyectos de investigación. Hay que tener en cuenta que no todos tienen las mismas habilidades para el desarrollo de sus investigaciones, por tanto, unos más que otros requerirán mayor asistencia técnica y seguimiento personalizado.

Asimismo, será fundamental avanzar en la creación de comunidades autocríticas de docentes en las instituciones formadoras donde laboramos, que nos permitan a través de un diálogo reflexivo y consensuado, dar respuesta a los problemas que se presentan en torno al desarrollo de los proyectos de investigación, y consolidar en el equipo habilidades y actitudes para la mejora constante de las prácticas docentes. Este reto implica, en primer lugar, comprender que la investigación-acción no es una tarea en solitario sino que exige el compromiso y esfuerzo sostenido de los diversos actores que participan en la comunidad educativa; y asimismo, poner en marcha estrategias concretas de sensibilización, motivación, y comunicación fluida entre quienes integran el equipo docente que tiene a su cargo el desarrollo de los proyectos de investigación.

Según Blández (2000, p. 25),

[...] la investigación-acción representa un gran antídoto contra la apatía y la desmotivación profesional, porque refuerza en sus participantes el interés por mejorar su práctica docente, su actitud abierta al cambio y su continuo compromiso con el proceso educativo, permitiéndoles mantener la ilusión por su labor educativa.

En ese sentido, en el marco del proceso de implementación del nuevo plan curricular en las carreras de Educación Inicial y Primaria nos sentimos cada vez más comprometidos con la tarea de crear las condiciones y estrategias que permitan consolidar la investigación-acción como parte constitutiva de nuestro Plan de Estudios y de nuestro quehacer docente, y de ese modo, facilitar la renovación constante de nuestras prácticas y de nuestro saber profesional. 


\section{REFERENCIAS BIBLIOGRÁFICAS}

Balcazar, F. (2003). Investigación acción participativa (iap): Aspectos conceptuales y dificultades de implementación. Fundamentos en Humanidades, IV(7-8), 59-77.

Blández Ángel, J. (2000). La investigación-acción: un reto para el profesorado. Guía práctica para grupos de trabajo, seminarios y equipos de investigación (2a ed.). Barcelona, España: INDES Publicaciones.

Carr, W. \& Kemis, S. (1988). Teoría crítica de la enseñanza. La investigación-acción en la formación del profesorado. Barcelona, España: Martínez Roca.

Elliot, J. (2010). La investigación-acción en educación ( $4^{\mathrm{a}}$ ed.). Madrid, España: Morata.

Kemmis, S. \& McTaggart, R. (1988). Cómo planificar la Investigación-Acción. Barcelona, España: Laertes.

Latorre, A. (2007). La investigación-acción: conocer y cambiar la práctica educativa. Barcelona, España: Graó.

Lewin, K. (1946). Action research and minority problems. Journal of Social Issues, 2(4), 34-46. https://doi.org/10.1111/j.1540-4560.1946.tb02295.x

Lomax, P. (1990). Managing Staff Development in Schools. Clevedon, Reino Unido: Multilingual Matters.

McKernan, J. (2001). Investigación-acción y curriculum: métodos y recursos para profesionales reflexivos. Madrid, España: Morata.

Pérez Serrano, G. (1990). Investigación-acción: aplicaciones al campo social y educativo. Madrid, España: Dykinson.

Restrepo Gómez, B. (2003). Aportes de la investigación-acción educativa a la hipótesis del maestro investigador. Pedagogía y Saberes, (18), 65-69. Recuperado de: http://www.pedagogica.edu.co/storage/ps/articulos/ pedysab18_09arti.pdf. https://doi.org/10.17227/01212494.18pys65.69

Suárez Pazos, M. (2002). Algunas reflexiones sobre la investigación-acción colaboradora en la educación. Revista Electrónica de Enseñanza de las Ciencias, 1(1), 1-17. Recuperado de: http://repositorio.minedu.gob.pe/bitstream/ handle/123456789/1835/Algunas\%20reflexiones\%20sobre\%20la\%20 investigacion-accion $\% 20$ colaboradora $\% 20 \mathrm{de} \% 201 \mathrm{a} \% 20$ educacion. pdf?sequence $=1$ \&isAllowed $=\mathrm{y}$ 